\title{
DESARROLLO INTENSIVO EN LA AGRICULTURA PARANAENSE. La sustentabilidad socioambiental jaqueada
}

\author{
Guillermo Foladori ${ }^{1}$ \\ Humberto Tomassino ${ }^{2}$
}

\author{
${ }^{1}$ Professor visitante do Programa de Doutorado em \\ Meio Ambiente e Desenvolvimento da Universidade Federal do Paraná. \\ ${ }^{2}$ Professor da Facultad de Veterinária da Universidad de la República, Uruguai; \\ Doutorando em Meio Ambiente e Desenvolvimento na Universidade Federal do Paraná.
}

\begin{abstract}
Neste artigo se analisam os dados dos Censos Agropecuários do Paraná desde 1970 a 1995. A partir dos mesmos, se caracteriza o desenvolvimento da agricultura parananense, e se conclui que o processo de tencnificação da agricultura conduz à insustentabilidade dos espaços rurais e urbanos em geral. Quanto ao espaço rural, é possivel verificar um processo acentuado de diferenciação social, que gera a proletarização dos pequenos produtores e a expulsão de uma importante fração da população rural. Além disso, gera um importante processo de degradação do recursos naturais. No ambiente urbano, o processo de tecnificação agrário acarreta importantes impactos ambientais indiretos, representados por uma intensa concentração de população desempregada ou sub-empregada, resultando em uma situação de insustentabilidade socioambiental crescente.
\end{abstract}

Palavras-chave: desenvolvimento agrário; diferenciação social; agricultura familiar.

$\mathbf{E}$ n los últimos meses de 1998 fueron divulgados los resultados del Censo Agropecuario de 1995 correspondiente al Estado de Paraná. Estos son de suma importancia para entender el proceso de desarrollo del capitalismo en la agricultura en este estado de la Región Sur del Brasil.

En este artículo vamos a mostrar algunas conclusiones del análisis de los datos de los Censos Agropecuarios de Paraná, así como reflexionar teóricamente sobre sus implicaciones. La conclusión más general es la confirmación de que a partir de la década de los ochenta, y más claramente entre 1985 y 1995 (último periodo intercensal) la agricultura paranaense se consolida en su fase de desarrollo intensivo, frente al periodo de transición que representó la década de los setenta. La fase intensiva va de la mano con la tendencia al despoblamiento absoluto del medio rural $y$, con ello, la creación de uno de los principales problemas ambientales del mundo actual: la concentración de la población en las ciudades. Toda fase de desarrollo del capitalismo en la agricultura genera desequilibrios socioambientales. Pero, la fase intensiva agudiza el proceso de diferenciación social y profundiza la tendencia al despoblamiento absoluto del campo. Desde el punto de vista ambiental el desarrollo intensivo tiene efectos inmediatos en la depredación y polución de los recursos; al introducir masivamente productos químicos poluyentes, agua de riego, mecanización, destrucción de ecosistemas por la especialización y monocultivo, factores todos que han sido ampliamente analizados. Pero, también tiene efectos de mayor alcance y fuera del área rural, al expulsar la población rural masivamente y generar, con ello la insustentabilidad urbana.

\section{EL DESARROLLO INTENSIVO Y EL DESPOBLAMIENTO ABSOLUTO DEL CAMPO}

La cantidad de predios ha disminuído notablemente en las últimas décadas. Para la Región Sur, y por Estados, el siguiente cuadro ilustra este hecho. 
Evolución de la cantidad de predios agropecuarios entre 1970 y 1995

\begin{tabular}{c|c|c|c|c|c}
\hline Estados & 1970 & 1980 & 1985 & 1995 & $\begin{array}{c}\text { Variación \% } \\
1970-1995\end{array}$ \\
\hline RS & 511650 & 474659 & 496579 & 429383 & $-16,1$ \\
Indice $1970=100$ & 100,0 & 92,8 & 97,1 & 83,9 & \\
SC & 207218 & 216159 & 234973 & 203347 & $-1,9$ \\
Indice $1970=100$ & 100,0 & 104,0 & 113,0 & 98,0 & \\
PR & 554453 & 453912 & 466235 & 369807 & $-33,3$ \\
Indice 1970=100 & 100,0 & 81,9 & 84,1 & 66,7 & \\
\hline Total & 1273321 & 1144730 & 1197787 & 1002537 & $-21,3$ \\
\hline
\end{tabular}

Fuente: Censos Agropecuarios. IBGE.

Para la Región Sur en su conjunto, los predios agropecuarios disminuyeron en un quinto, y Paraná tuvo el índice de reducción más significativo de la región, viendo desaparecer cerca de 180 mil predios en una generación, un tercio de lo que había en 1970.

Si consideramos que la superficie total en explotación ha venido aumentando, la disminución de la cantidad de predios sólo puede ser resultado de una concentración y centralización de la propiedad agropecuaria en cada vez menos manos. ${ }^{1}$ Concentración en cuanto menos productores son dueños de cada vez más superficie de tierras. Centralización porque las tierras con destino productivo agropecuario tienden a unificarse en unidades compactas de producción, lo cual significa economías de escala para sus dueños.

La concentración y centralización de la propiedad no es algo novedoso. No obstante, la concentración y centralización del suelo que se destina a uso agropecuario adquiere una dimensión diferente. Mientras en la industria la concentración de las empresas y el capital puede ir ligada - y normalmente así ocurre - a una también concentración de la población, ya que las áreas urbanas son simultáneamente centros de producción industrial y centros de habitación humana, en la agricultura la concentración del suelo y el capital va ligado a un despoblamiento absoluto del campo.

En las áreas urbanas el incremento del capital industrial y el desarrollo tecnológico desplazan fuerza de trabajo que permanece habitando la ciudad, sea como empleados en otras industrias o sectores de la economía, sea inclusive como desempleados. La ciudad es simultáneamente asiento de producción industrial, como asiento de habitación y vida. Por ello, el desarrollo industrial desplaza fuerza de trabajo en términos relativos: de unas industrias para otras, o de unos barrios para otros. Por el contrario, en las áreas rurales todo incremento del capital y desarrollo tecnológico se traduce en "limpiar" el campo de gente, porque se requiere el suelo como objeto de trabajo. La tierra es medio de producción. Pero, hay que tener claro que, esta característica del desarrollo del capitalismo en la agricultura sólo se presenta en su fase más desarrollada, en la etapa donde predomina el desarrollo intensivo. Por el contrario, en los primeros momentos, cuando predomina el desarrollo extensivo puede, perfectamente, ocurrir lo contrario, es decir, un aumento de la población.

Si consideramos las fases del desarrollo del capitalismo en la agricultura, podemos distinguir, claramente, una primera, de avance de la producción capitalista en amplitud. Este desarrollo extensivo se caracteriza por la introducción de relaciones capitalistas al interior de formas mercantil simples de producción; o bien la colonización de nuevas áreas. ${ }^{2}$ Durante estos primeros momentos, la producción capitalista puede contratar trabajo asalariado llegando inclusive a aumentar el número de personas viviendo en el medio rural.

Tan pronto el capital introduce la mecanización en la agricultura de forma sostenida, surge una segunda fase, de desarrollo del capitalismo en intensidad. Esto significa la inversión de cada vez mayor volúmen de capital sobre la misma extensión de suelo. En esta fase pueden apreciarse efecto sociales y directamente ambientales. ${ }^{3}$ La teoría de la renta capitalista del suelo explica cómo las inversiones intensivas de capital sobre el mismo suelo pueden llevar a mayores ganancias para los empresarios simultáneamente con un deterioro creciente de la riqueza natural. De manera que desarrollo intensivo y sustentabilidad medio-ambiental suelen manifestarse como opuestos. ${ }^{4}$ Desde el punto de vista de la población ocurren dos grandes consecuencias. Por un lado, se desplaza fuerza de trabajo en términos absolutos; por otro, acontece la ruina acelerada de las explotaciones de producción mercantil simple, que no pueden soportar la competencia impuesta por los costos de producción de la agricultura mecanizada capitalista.

Esta distinción de las diferentes fases del desarrollo del capitalismo en la agricultura es de fundamental importancia para no caer en posiciones equivocadas, de pensar que las explotaciones capitalistas son capaces de desplazar a la producción mercantil simple en cualquier fase o, por el contrario, suponer que la producción mercantil simple sobrevive aún en la fase de mecanización agrícola sostenida. ${ }^{5}$

Ambas fases, la extensiva y la intensiva, se dan combinadas. No obstante, en cada momento es posible distinguir cuál predomina. En Paraná, los datos extraídos de los Censos Agropecuarios muestran una fase de transición del desarrollo del capitalismo en extensión para uno intensivo en el periodo que va de 1970 a 1980 . En ese periodo continúa creciendo la superficie en explotación, a una tasa anual media de $2,4 \%$ (2,3\% en $1970-75$; y $5,1 \%$ entre 1975 y 1980$)$. No obstante, es también la década en que la mecanización de la agricultura paranaense crece al mayor ritmo (19\% anual en el primer quinquenio de los setenta, y $7 \%$ en el segundo).

A partir de 1985 es claro el predominio del crecimiento en intensidad sobre la modalidad expansiva. Por un lado, la superficie en explotación se estanca. Por otro, la mecanización continúa creciendo a tasas menores pero significativas. El cuadro que sigue muestra la cantidad de tractores existentes cada 100 hectáreas de tierras cultivadas durante los 25 años intercensales. 
Paraná. Superficie cultivada y utilización de tractores

\begin{tabular}{c|r|r|r|r|c}
\hline Años & \multicolumn{1}{c|}{$\begin{array}{c}\text { Tierras } \\
\text { cultivadas** } \\
\text { (miles de } \\
\text { hectáreas) }\end{array}$} & $\begin{array}{c}\text { Tractores } \\
\text { (cantidad) }\end{array}$ & $\begin{array}{l}\text { Hectáreas } \\
\text { por tractor }\end{array}$ & $\begin{array}{l}\text { Tractores } \\
\text { cada 100 } \\
\text { hectáreas }\end{array}$ & $\begin{array}{c}\text { Tasa anual media } \\
\text { de crecimiento de } \\
\text { tractores cada 100 } \\
\text { hectáreas (\%) }\end{array}$ \\
\hline 1970 & 7418 & 18619 & 398 & 0,25 & - \\
1975 & 8927 & 52498 & 170 & 0,59 & 18,8 \\
1980 & 10071 & 81727 & 123 & 0,81 & 6,5 \\
1985 & 10639 & 101346 & 81 & 0,95 & 3,2 \\
1995 & 10400 & 130828 & 79 & 1,26 & 2,9 \\
\hline
\end{tabular}

Fuente: Censos Agropecuarios. IBGE

* Cultivos permanentes + temporales + pastos sembrados.

Vale la pena anotar que durante un sólo quinquenio, el primero de los setenta, la agricultura paranaense pasa, de un nivel de mecanización semejante a los países más atrasados de América Latina, a casi alcanzar la media de Argentina. 15 años después, en 1985 ya estaba al nivel de Argentina y Uruguay, países estos donde la mecanización se introdujo tempranamente. Es claro que el Paraná extractivista termina a comienzos de los setenta; y el Paraná de agricultura intensiva se establece firmemente a mediados de los ochenta.

Las consecuencias de esta fase intensiva en cuanto a la disminución de la ocupación y la tendencia al despoblamiento absoluto del campo son nítidas. Veamos los datos del cuadro siguiente,

Personal ocupado en los establecimientos agropecuarios. Paraná. 1970-1995

\begin{tabular}{|c|c|c|}
\hline Años & $\begin{array}{l}\text { Personal ocupado } \\
\text { (miles de personas) }\end{array}$ & $\begin{array}{c}\text { Tasa media anual } \\
\text { de crecimiento }(\%)\end{array}$ \\
\hline 1970 & 1981 & - \\
\hline 1975 & 2079 & 1,0 \\
\hline 1980 & 1808 & $-2,8$ \\
\hline 1985 & 1855 & 0,5 \\
\hline 1995 & 1288 & $-3,6$ \\
\hline
\end{tabular}

Fuente: Censos Agropecuarios. IBGE.

Puede verse cómo el personal ocupado en los establecimientos disminuye en forma absoluta, primero en el quinquenio $1975-80$, a una tasa negativa de $2,8 \%$, y luego de una mínima recuperación que no alcanza ni al crecimiento natural de la población durante el quinquenio 1980-85, comienza a disminuir de manera contundente a una tasa negativa de 3,6\% anual en el decenio 1985-95. El resultado es la desaparición de más de medio millón de personas ocupadas en tareas agropecuarias en 10 años.

Esta conclusión debe complementarse con la brindada por los Censos Demográficos, que ya muestran una disminución absoluta de la población rural desde la década de los setenta, según el cuadro que sigue,

Población urbana y rural. Paraná (miles de personas)

\begin{tabular}{c|c|c|c|c|c}
\hline Años & $\begin{array}{c}\text { Población } \\
\text { total } \\
\text { (residentes) }\end{array}$ & $\begin{array}{c}\text { Población } \\
\text { urbana }\end{array}$ & $\begin{array}{c}\text { Población } \\
\text { rural }\end{array}$ & $\begin{array}{c}\text { Tasa media } \\
\text { anual de cre- } \\
\text { cimiento (\%) }\end{array}$ & $\begin{array}{c}\text { Población } \\
\text { urbana (\%) }\end{array}$ \\
\hline 1970 & 6930 & 2504 & 4425 & - & 36,0 \\
1980 & 7629 & 4473 & 3157 & $-3,3$ & 41,0 \\
1991 & 8449 & 6198 & 2251 & $-3,0$ & 73,0 \\
1996 & 9004 & 7012 & 1992 & $-2,4$ & 78,0 \\
\hline
\end{tabular}

Fuente: Censos Demográficos. IBGE
Es claro que a medida que aumenta la población urbana en detrimento de la rural, las tasas de decrecimiento de la población rural tienden a disminuir. Para 1996, casi el 80\% de la población de Paraná reside en ciudades o villas.

Los resultados de Paraná se corresponden con lo que ocurre en los otros dos estados de la Región Sur. En los 15 años entre 1980 y 1995 el personal ocupado en la agricultura disminuye en más de un $20 \%$, y Paraná tiene el porcentaje mayor, con casi un $29 \%$.

Personal ocupado. Región Sur. 1980 a 1995

\begin{tabular}{c|r|r|r|r}
\hline Estados & 1980 & 1985 & 1995 & $\begin{array}{c}\text { Variación \% } \\
(1980-1995)\end{array}$ \\
\hline RS & 1746540 & 1747235 & 1376208 & $-21,2$ \\
SC & 836755 & 887287 & 718694 & $-14,1$ \\
PR & 1807328 & 1854836 & 1287520 & $-28,8$ \\
Total & 3553868 & 3602071 & 2663728 & $-25,0$ \\
\hline
\end{tabular}

Fuente: Censos Agropecuarios. IBGE.

En una generación, en 25 años, la mecanización e inversión de capital en la agricultura paranaense desmontó la antigua estructura extractivista y, también, "limpió" la tierra de buena parte de la pequeña producción.

Esta dinámica del capitalismo en el campo genera, como contraparte, consecuencias urbanas. La Región Metropolitana de Curitiba tuvo la mayor tasa de crecimiento de las regiones metropolitanas de Brasil en la década de 1970 $(5,79 \%)$, y el tercer lugar en la década de los ochenta $(3,02 \%$, luego de Fortaleza y Salvador). Para 1995, casi el 30\% de la población residente en la Región Metropolitana de Curitiba era migrante. ${ }^{6}$ Los problemas de empleo, vivienda, salud y educación, así como los de polución y depredación de paisajes, ligados a estos rápidos ritmos de crecimiento son comunes a las grandes ciudades. Considerando los datos hasta 1991, la Región Metropolitana de Curitiba ha tenido un desempeño relativamente más feliz que las otras regiones metropolitanas del Brasil en lo que a la pobreza se refiere. Según diferentes estimaciones, el porcentaje de pobres está entre $8,1 \%$ (estimación de Albunquerque, 1993), y 17,4\% (estimación de Sabóia, 1991), para 1991. La región metropolitana que le sigue es la de Porto Alegre con casi el doble (dependiendo de la estimación). Esta ventaja relativa de la Región Metropolitana de Curitiba parece explicarse por dos razones interconectadas. Por un lado, porque La Región Metropolitana de Curitiba contiene una importante actividad rural en pequeñas unidades de producción, que logran elevar su nivel de vida frente a las actividades exclusivamente urbanas. Por otro lado, porque el empleo industrial de Curitiba durante el periodo 1970/1991 fue el segundo mayor del país, con una tasa media anual de crecimiento de $6 \%$, mayor al crecimiento de la población. Inclusive en el periodo 1980/91, la tasa de empleo industrial fue de $3,5 \%$ anual, superior también a la del crecimiento de la población (Delgado, 1997). A pesar de esta situación relativamente mejor, si consideramos el importante volúmen aún existente de predios agropecuarios, así como el el ritmo de disminución y la fase claramente intensiva del desarrollo del capitalismo en la agricultura, las perspectivas para las proximas décadas no parecen ser en nada halagüieñas. 


\section{LA DIFERENCIACIÓN SOCIAL Y LA DESAPARICIÓN DE LA PRO- DUCCIÓN MERCANTIL SIMPLE}

El desarrollo intensivo implica la polarización más profunda de la población ocupada en la agricultura. En un primer momento, crece el trabajo asalariado en detrimento de los productores directos; luego disminuye también el trabajo asalariado, como resultado de su desplazamiento por la mecanización agrícola. La polarización se completa con la concentración y centralización del suelo en pocas manos.

Si prestamos atención a los predios según grupos de tamaño de superficie, y aunque el tamaño de superficie no es de manera alguna el indicador principal, el resultado es a todas luces contundente: mientras por un lado asistimos a una fuerte concentración de la propiedad del suelo, por otro asistimos a una fuerte proletarización de los productores directos. El cuadro a continuación muestra la relación entre número de predios y superficie por grupos de estratos.

Evolución del número de predios por grupos de estratos de tamaño de tierra entre 1970 y 1995 . Paraná

\begin{tabular}{|c|c|c|c|c|c|c|c|}
\hline 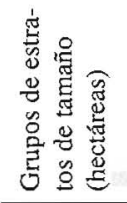 & $\stackrel{\circ}{\stackrel{2}{2}}$ & \begin{tabular}{l}
$\infty$ \\
$\Omega$ \\
\hdashline
\end{tabular} & $\begin{array}{l}n \\
\infty \\
=\end{array}$ & $\stackrel{2}{2}$ & 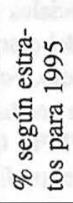 & 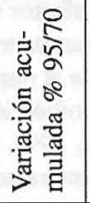 & 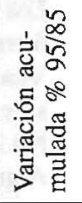 \\
\hline $\begin{array}{l}\text { menos } \\
\text { de } 10\end{array}$ & 295272 & 214995 & 229015 & 154620 & 42,0 & $-47,63$ & $-32,48$ \\
\hline $\begin{array}{l}10 \text { a menos } \\
\text { de } 50\end{array}$ & 218625 & 189900 & 186718 & 163078 & 44,0 & $-25,41$ & $-12,66$ \\
\hline $\begin{array}{l}50 \text { a menos } \\
\text { de } 100\end{array}$ & 22311 & 25131 & 25529 & 25227 & 7,0 & 13,07 & $-1,18$ \\
\hline 100 y más & 18245 & 23886 & 24973 & 26872 & 7,0 & 47,28 & 7,60 \\
\hline Total & 554453 & 453912 & 466235 & 369807 & 100,0 & $-33,30$ & $-20,68$ \\
\hline
\end{tabular}

Fuente: tomado del Boletim de Deser, Setembro 1998, Curitiba.

Si existía alguna duda hasta el Censo Agropecuario de 1985, respecto al proceso de diferenciación social y exclusión de los pequeños productores mercantiles, los datos del Censo Agropecuario de 1995 terminan con ella. En el último periodo intercensal la cantidad total de predios disminuye una quinta parte, pero no todos los estratos lo hacen por igual. El estrato de menos de 10 hectáreas, donde debe concentrarse la mayoría de los pequeños productores directos sufre la mayor reducción, en un tercio. Sigue en disminución el estrato de 10 a menos de 50 , un estrato que podría considerarse también de pequeños productores directos, dependiendo del destino productivo principal y tipo de tierras. Este estrato de 10 a menos de 50 sufre una reducción del $13 \%$ en el último periodo intercensal, aunque se redujo en una cuarta parte si tomamos el periodo entre 1970 y 1995 . Sin embargo, cuando pasamos a los estratos de productores con mayor superficie, donde ciertamente se encuentran no sólo productores acomodados, sino la mayoría de las empresas agropecuarias capitalistas, vemos un crecimiento. ${ }^{8}$
El análisis de los predios por tamaño de superficie es sólo una gruesa aproximación, pero puede resultar engañoso al llevar a pensar que las diferencias son sólo cuantitativas, cuando no es así. Existen notorias diferencias en cuanto al lugar que ocupan los representantes del primer estrato de menos de 10 hectáreas en la trama de las relaciones de producción. No sería aventurado aseverar que, la mayoría de este estrato más que productores directos son en realidad verdaderos semiproletarios con parcela. ${ }^{9}$

Para sustentar la sospecha anterior, basta ver los datos sobre la disminución de los responsables y familiares en el total del personal ocupado en los establecimientos. El cuadro que sigue es contundente al respecto.

Personal ocupado según responsables y familiares y personal contratado. Paraná 1970-1995 (miles de personas)

\begin{tabular}{l|l|c|c|c}
\hline Años & $\begin{array}{c}\text { Personal } \\
\text { ocupado }\end{array}$ & $\begin{array}{c}\text { Responsables } \\
\text { y familiares }\end{array}$ & Contratados & $\begin{array}{c}\text { \% responsables } \\
\text { y familiares }\end{array}$ \\
\hline 1970 & 1981 & 1696 & 285 & 85,6 \\
1975 & 2079 & 1674 & 405 & 80,5 \\
1980 & 1808 & 1369 & 439 & 75,7 \\
1985 & 1855 & 1375 & 480 & 74,1 \\
1995 & 1288 & 853 & 435 & 66,2 \\
\hline
\end{tabular}

Fuente: Censos Agropecuarios. IBGE.

El cuadro indica la reducción de todo el personal ocupado en la agricultura, como habíamos señalado anteriormente, pero también la reducción aún más fuerte de los responsables y familiares, lo que demuestra un retroceso del trabajo directo frente a un beneficio relativo del trabajo asalariado. ${ }^{10}$ Este último también decrece en el último periodo intercensal, como resultado del desarrollo intensivo ligado a la mecanización y mayor inversión de capital por unidad de superficie.

Si alguna ventaja podía tener el productor directo frente a la empresa capitalista, ésta era la posibilidad de producir directamente el alimento, con lo cual el pequeño productor garantía su reproducción mínima. La expansión y profundización de las relaciones mercantiles a aquellos productos con importante componente de autoconsumo es un doble indicador, tanto del retroceso de la producción directa frente a la capitalista, como del hecho de que la pequeña producción mercantil no es una forma de producción enfrentada a la capitalista, sino que se rige por las mismas leyes del mercado. ${ }^{11}$ En el cuadro que sigue hemos seleccionado los dos productos con mayor componente de autoconsumo, para ver la evolución de su destino en los últimos veinte años.

Producción total y consumo en el establecimiento de maíz y mandioca. Paraná. 1975-1995 (miles de toneladas)

\begin{tabular}{|c|c|c|c|c|c|c|}
\hline 号 & 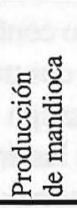 & 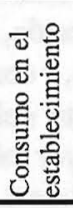 & 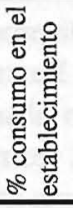 & 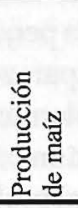 & 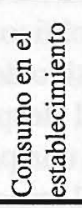 & 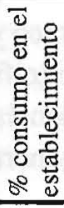 \\
\hline 1975 & 347 & 256 & 74,0 & 3430 & 835 & 24,0 \\
\hline 1980 & 685 & 533 & 78,0 & 3908 & 1062 & 27,0 \\
\hline 1985 & 930 & 469 & 50,0 & 4151 & 784 & 19,0 \\
\hline 1995 & 1438 & 388 & 27,0 & 6598 & 911 & 14,0 \\
\hline
\end{tabular}

Fuente: Censos Agropecuarios. IBGE. 
En la mandioca, el consumo en el establecimiento pasa de ser un $74 \%$ en 1975 a ser el $27 \%$ en 1995 . En lo que respecta al maíz, la reducción pasa del $24 \%$ al $14 \%$ en el mismo periodo. La conclusión de estos datos muestra que la pequeña producción ha profundizado su destino mercantil en detrimento del autoconsumo, con ello la vulnerabilidad frente a la producción capitalista aumenta.

Pero, si la tendencia al despoblamiento absoluto del campo paranaense resulta clara del análisis expuesto, no hay que perder de vista que se trata de un proceso. Aún existen cerca de 370 mil predios, de los cuales mas del $80 \%$ tienen menos de 50 hectáreas. Es previsible, salvo políticas radicales de reforma agraria, o fuerte apoyo a la pequeña producción, que la migración campo-ciudad continúe en las próximas décadas, a los ritmos mostrados por la pérdida de ocupación agropecuaria anotados. Independientemente de los esfuerzos que técnicamente puedan realizarse para superar los problemas de polución por tóxicos en la agricultura paranaense, o de depredación de ecosistemas, seres vivos, y agua, el hecho es que las relaciones capitalistas crean con su propio desarrollo, y aunque pasen de una fase a otra, problemas ambientales cada vez más difíciles de solucionar. Al desplazar población del campo a la ciudad, al agudizar la contradicción campo/ciudad, traslada la crisis ambiental para dentro de las propias ciudades.

\section{CONCLUSIONES}

El desarrollo sustentable en Paraná está doblemente jaqueado. A nivel rural el desarrollo intensivo del capital en la agricultura genera procesos insustentables desde una óptica ambiental, ya que degrada y poluye recursos naturales. Desde una óptica ocial, provoca procesos de empobrecimiento y expulsión de población. A nivel urbano, por su parte, acumula población migrante, desempleada o subempleada, y disminuye aceleradamente la calidad de vida de las grandes ciudades.

Según los datos analizados de los Censos Agropecuarios, la agricultura paranaense ha entrado, a partir de la década de los ochenta en su fase de desarrollo intensivo. Esta, a diferencia de la fase extensiva, tiene dos características fundamentales. Por un lado, las empresas capitalistas ya mecanizadas compiten ventajosamente frente a la pequeña producción mercantil. La consecuencia es un proceso de proletarización y ruina de esta pequeña producción. Como el número de predios en la agricultura de Paraná es aún significativo, es de esperar que en las próximas décadas, de no haber una política radical de reforma agraria, o fuerte apoyo económico y político a la pequeña producción, otro contingente muy significativo desaparezca. Por otro, y como contracara, la tendencia al despoblamiento absoluto del campo paranaense, generará una presión cada vez mayor sobre las áreas urbanas.

El principal problema ambiental generado por el desarrollo intensivo del capitalismo en la agricultura no es la depredación del suelo, ni los altos niveles de polución resultado del uso de tóxicos químicos. Estos problemas que ciertamente aumentan, resultan insignificantes frente a la profundización de la división campo/ciudad, y al crecimiento urbano. Cuando los más modernos estudios sobre el desarrollo agrario llaman la atención sobre la necesidad de conservar los recursos, y se autodenominan de "manejo de recursos" (resource management), ${ }^{12}$ olvidan que las leyes del desarrollo del capitalismo deben ser contempladas a nivel global, y que las alternativas técnicas de desarrollo sustentable siempre están subordinadas a la dinámica que las relaciones sociales les imprimen.

\section{NOTAS}

1 La superficie aprovechada entre 1970 y 1995 tiende a aumentar en Paraná, como se deriva del cuadro siguiente:

\begin{tabular}{c|c|c|c}
\hline Años & $\begin{array}{c}\text { Tierras aprovechadas } \\
\text { (miles de hectáreas) }\end{array}$ & $\begin{array}{c}\text { Indice } \\
1970=100\end{array}$ & $\begin{array}{c}\text { Tasa media anual } \\
\text { de crecimiento (\%) }\end{array}$ \\
\hline 1970 & 11797 & 100 & - \\
1975 & 13206 & 112 & 2,3 \\
1980 & 14902 & 126 & 5,1 \\
1985 & 15499 & 131 & 0,8 \\
1995 & 14964 & 127 & $-0,4$ \\
\hline
\end{tabular}

Fuente: Elaboración propia a partir de los Censos Agropecuarios. IBGE.

2 En la nota anterior puede verse un significativo crecimiento de la superficie en explotación durante la década de los setenta, y un estancamiento durante los ochenta y noventa.

3 Los efectos sociales también son ambientales. Los congéneres humanos forman parte del entorno.

4 Véase al respecto, Foladori, G, Tomassino, H, "La degradación del suelo: su explicación mediante la teoría de la renta". Revista de Geografia. No.2, 1999. UFPR. Curitiba.

5 Algunos autores prefieren llamar a ésta de "economía familiar". La mayoría de los equívocos en relación a la polémica sobre la viabilidad de una economía familiar en un medio capitalista, radican en desconocer que la economía familiar, de producción mercantil simple, economía campesina, economía de la pequeña producción, según se le llame, en la medida en que produce para el mercado se rige por la ley del valor al igual que la economía capitalista. No se trata de una economía diferente, enfrentada a la dinámica del capital, sino de formas de producción ya mercantiles, cuyo desarrollo lleva, naturalmente, a la producción capitalista.

6 Migrantes con hasta 15 años de residencia. Pero, prácticamente la mitad de ellos llegó en la década de los 90. (Delgado, 1997:8).

7 En un artículo sobre la agricultura en el sur del Brasil, publicado en 1997, se dice:

"O êxodo rural (Deser, 1996) apesar de menos intenso, é ainda significativo, principalmente no Paraná, que apresenta o maior índice de êxodo do país. Neste estado, nas duas últimas décadas, cerca de 2.600 .000 pessoas saíram de seu local de origem, das quais cerca de 1.400.000 dirigiram-se às cidades e as demais 1.200 .000 migraram para outras terras agrícolas. Ao mesmo tempo, constata-se que estão se tornando cada vez mais pobres, alguns chegando ao limite da indigência e da miséria. Segundo o Mapa da Fome (Peliano, 1993), 32 milhões de brasileiros passam fome, o que representa $22 \%$ da população total. Na região Sul há 4.082 .313 indigentes, dos quais quase $60 \%$ residem no meio rural, sendo $59,91 \%$ no Paraná, 63,64\% em Santa Catarina e 57,07\% no Rio Grande do Sul (Pnad/ IBGE, 1990 apud Deser, 1994:24).

No Estado do Paraná, as consequências do processo de modernização da agricultura foi intensivo, gerando riquezas, mas também proporcionando os mais altos índices de concentração da renda agrícola (Veiga, 1985). A pobreza rural é marcante no estado, que se destacou como o quinto do país em número de indigentes na zona rural, acima de 1.000 .000 de pessoas, em 1990 (Peliano, 1993)" (Gehlen, de Mélo, 1997).

8 Para no recargar el cuadro no se desagregó el tamaño de 100 hectáreas y más. Es conveniente advertir que en el estrato de más de 10000 hectáreas, hubo un retroceso aún más acentuado que en el de menos de 10 hectáreas, tanto en el periodo 1970-95, como en el 1985-95.

- El Boletim do Deser de Setembro de 1998 advierte que por cambios en la fecha de recolección de la información del Censo Agropecuario de 1995, respecto de los anteriores, "...deixou de encontrar uma parcela de pequenos 
agricultores, principalmente parceiros e arrendatários, que não se encontravam físicamente no local de trabalho neste período", lo que puede tomarse como una confirmación de su carácter de semiproletarios.

${ }^{10}$ Uno de los argumentos que se esgrimen en favor de políticas dirigidas a la pequeña producción es que buena parte de la producción de importantes rubros agrícolas están, a pesar de esta disminución, mayoritariamente en manos de pequeños productores. Creemos que el razonamiento es equivocado tanto por la justificativa, como por el resultado. Si la defensa de la pequeña producción se basa en el hecho de que producen buena parte de ciertos rubros agropecuarios, una vez que esto deje de ocurrir no habría argumento alguno. Por el contrario, el argumento debe radicar en que aún constituye uno de los medios más simples y profundos para reducir el desempleo, al tiempo que a través de determinadas formas de organización del trabajo permiten la elevación de la conciencia política. Por otra parte, es discutible que aquellos rubros sean realmente resultado de lo que podríamos llamar producción directa o economía familiar. En el caso del tabaco, suinos, la carne de ave, los huevos, o el tomate, la mayoría de sus productores podrían considerarse "asalariados a domicilio", ya que sus posibilidades de "control" o "decisión" sobre el producto del trabajo son mínimas, cuando no se reducen exclusivamente al riesgo. Si algún sentido tiene el concepto de productor directo o economía familiar, éste radica en el control del proceso productivo. Cuando este proceso es dictaminado por el capital que le "adelanta" el crédito, los insumos, las semillas o el pie de planta, y tiene el monopolio sobre la compra, no existe control alguno.

"La denominación "economía mercantil simple" y "economía mercantil ampliada o capitalista" es más adecuada que "economía familiar" y "economía capitalista". En esta última pareja se pierde la identidad central de ambas: el carácter mercantil de la producción.

12 "The key question to be addressed shifts from: How do I improve the productivity of my farm?, to: How do I maintain the productivity of the natural resources on which my farming depends? (Jiggins, 1993:620).

\section{REFERÊNCIAS BIBLIOGRÁFICAS}

DELGADO, Paulo Roberto. Pobreza e meio ambiente na Região Metropolitana de Curitiba. Mimeo, Doutorado em Meio Ambiente e Desenvolvimento, UFPR. Curitiba, 1997.

DESSER. Boletim do Desser. Cojuntura Agrícola. Agosto, 1998.

FOLADORI, Guillermo; TOMASSINO, Humberto. "La degradación del suelo: su explicación mediante la teoría de la renta". Revista de Geografia. No.2, 1999. UFPR. Curitiba.

GEHLEN, Ivaldo; DE MÉLO, José. "A dinâmica da agricultura no sul do Brasil. Realidade e perspectivas". São Paulo em Perspectiva. Vol. 11 No. 2.Fundação SEADE, São Paulo, 1997.

IBGE (Instituto Brasileiro de Geografia e Estatística). Censos Agropecuarios. Varios años.

Censos Demográficos. Varios años.

JIGGINS, Janice. "From technoly transfer to resource management". Proceedings of the DVII International Grassland Congress, 1993. 